BNL-114128-2017-JA

\title{
A Bayesian Approach Based Outage Prediction in Electric Utility Systems Using Radar Measurement Data
}

\author{
M. Yue, T. Toto, M. P. Jensen, S. E. Giangrande, and R. Lofaro
}

Submitted to IEEE Transactions on Smart Grid

July 2017

Sustainable Energy Technologies Department

Brookhaven National Laboratory

U.S. Department of Energy
USDOE Office of Science (SC)

New York State Energy Research and Development Authority (NYSERDA)

Notice: This manuscript has been authored by employees of Brookhaven Science Associates, LLC under Contract No. DE- SC0012704 with the U.S. Department of Energy. The publisher by accepting the manuscript for publication acknowledges that the United States Government retains a non-exclusive, paid-up, irrevocable, world-wide license to publish or reproduce the published form of this manuscript, or allow others to do so, for United States Government purposes. 


\section{DISCLAIMER}

This report was prepared as an account of work sponsored by an agency of the United States Government. Neither the United States Government nor any agency thereof, nor any of their employees, nor any of their contractors, subcontractors, or their employees, makes any warranty, express or implied, or assumes any legal liability or responsibility for the accuracy, completeness, or any third party's use or the results of such use of any information, apparatus, product, or process disclosed, or represents that its use would not infringe privately owned rights. Reference herein to any specific commercial product, process, or service by trade name, trademark, manufacturer, or otherwise, does not necessarily constitute or imply its endorsement, recommendation, or favoring by the United States Government or any agency thereof or its contractors or subcontractors. The views and opinions of authors expressed herein do not necessarily state or reflect those of the United States Government or any agency thereof. 


\title{
A Bayesian Approach Based Outage Prediction in Electric Utility Systems Using Radar Measurement Data
}

\author{
Meng Yue, Member, IEEE, Tami Toto, Michael P. Jensen, Scott E. Giangrande, and Robert Lofaro
}

\begin{abstract}
Severe weather events such as strong thunderstorms are some of the most significant and frequent threats to the electrical grid infrastructure. Outages resulting from storms can be very costly. While some tools are available to utilities to predict storm occurrences and damage, they are typically very crude and provide little means of facilitating restoration efforts. This study developed a methodology to use historical high-resolution (both temporal and spatial) radar observations of storm characteristics and outage information to develop weather condition dependent failure rate models (FRMs) for different grid components. Such models can provide an estimation or prediction of the outage numbers in small areas of a utility's service territory once the real-time measurement or forecasted data of weather conditions become available as the input to the models. Considering the potential value provided by real-time outages reported, a Bayesian outage prediction (BOP) algorithm is proposed to account for both strength and uncertainties of the reported outages and failure rate models. The potential benefit of this outage prediction scheme is illustrated in this study.
\end{abstract}

Index Terms - Radar observation, generalized linear model (GLM), regression analysis, failure rate, Bayesian update.

\section{INTRODUCTION}

$\mathrm{T}$ HERE exist a large number of studies attempting to develop models for damage assessments of the power grid under various severe external events, especially weatherrelated events, e.g., [1-12]. The majority of these models were developed based on statistical analyses of both historical power outage data and a variety of environmental and meteorological data of different spatial-temporal resolutions [1]. While some studies tried to estimate or predict weather event induced power outages directly [2-6], failure rate-based models [7-10] appear to be a very natural and popular option because the grid components are continuously operating and external severe weather events directly impact the failure rates. A number of failure rate models (FRMs) were developed

This work was partially supported by the New York State Energy Research and Development Agency (NYSERDA) under Grant 41310.

M. Yue and R. Lofaro are with the Department of Sustainable Energy Technologies, Brookhaven National Laboratory, Upton, NY 11973-5000 USA (e-mail: yuemeng@bnl.gov and lofaro@bnl.gov).

T. Toto, M. P. Jensen and S. E. Giangrande are with the Department of Environmental and Climate Sciences, Brookhaven National Laboratory, Upton, NY 11973-5000 USA (e-mail: ttoto@bnl.gov, mjensen@bnl.gov, sgrande@bnl.gov). based on different techniques such as regression analysis [7$10]$ and fuzzy sets theory [9].

The meteorological data used in previous studies are often spatially and temporally sparse, and rely on elementary model applications rather than taking advantage of publicly available observational data and advanced numerical weather prediction model output. In [2], observations such as one-minute wind gust speed measured at sparsely distributed surface-based weather stations were used to describe the meteorological conditions and develop county level fragility curves. In [3], power outages were directly estimated from Poisson and negative binomial generalized linear models (GLMs) based on data including weather model estimates of the maximum 3second gust wind speed and the length of time that the winds were above $20 \mathrm{~m} / \mathrm{s}$ in each grid cell (3.66 km by $2.44 \mathrm{~km})$ in the service area. Data with a similar resolution and a random forecast method were used in $[4,5]$ to estimate outages directly. The authors in [6] proposed a fuzzy logic approach for assessment of distribution system outages under hurricanes. Weather data were used to evaluate the weather related hazard and its impact on the probability of component outage occurrences. Wind speed was categorized into 5-classes and 15-levels scale using boundary values [6].

Very coarse weather measurement data, e.g., average wind speed during the storm or bulk categories of weather types were used to calculate failure rates based on multiple linear regression (MLR) [7] and GLMs [8], respectively. In [9], the impact of hurricanes (characterized by wind speed and rainfall) on the failure rates of transmission lines were modeled using a "Fuzzy Inference System" (FIS) based on a fuzzy clustering method. The spatial and temporal granularities of weather data were not specified but are expected to be low, as can be seen from the input data used in the case study [9].

The above methods are mainly used for long-term planning or post weather event assessment but not suitable for shortterm or online outage estimation/prediction, which will enhance the situational awareness during a severe weather event to facilitate distribution system restoration. Regarding short-term or online outage forecasting, the study in [10] used curve-fitting and real-time reported outage information, requiring accurate outage information. However, the major challenges faced by the existing Outage Management Systems (OMSs) include the lack of a reliable and timely outage 
reporting system and difficulty in identifying so-called "nested outages." A unique feature of distribution system outages is nested outages [11] resulting from the dominant radial configuration of the distribution feeders, e.g., two or more breaks of lines in a radial feeder with one below (or "nested" within) another. It is very difficult, if not impossible to identify downstream outages before the upstream outages are identified and fixed, and the feeder is energized, given the existing monitoring and communication capabilities possessed by most of the distribution utilities. Therefore, there are strong arguments that the reported outage information currently cannot play a dominant role in outage prediction.

The purpose of this study is to develop an improved outage prediction and estimation scheme by deriving failure rate models (FRMs) for grid components using weather radar observations of storm characteristics and grid component outage data; both at a very high level of granularity. The FRMs can be used for assessing severe weather impacts in both planning and operation, with applications, in this study, focusing on short-term forecasting and outage estimation for given radar measurements of weather conditions. The major difference between the FRMs developed here and the existing ones is the use of very high temporal and spatial resolution weather radar measurements, taking into account the "dynamic" time evolution of the weather condition, i.e., the impact is not only determined by the magnitude of weather conditions such as wind speed and radar reflectivity, but also the duration of these magnitudes during the storm. Therefore, the developed FRMs are expected to better capture the correlation between failures of different components and weather conditions, and be used for outage estimation and prediction with higher levels of granularity.

An FRM can be used alone for short-term outage prediction and estimation. However, the FRM models (1) work better for severe storms that may cause many outages and for longer term prediction due to their statistical nature; (2) do not deal with the inherent uncertainties of weather forecasting; and (3) do not use known outage information. To address these issues, a novel Bayesian outage prediction (BOP) algorithm is developed to account for both the strength and uncertainties of the reported outage and failure rate models. That is, the outages calculated based on failure rate models are used as prior information, and the reported outage as new evidence to update the posterior information to predict outages within the forecasting time window.

The approach is expected to be portable as the failure rates are developed for generic grid components, and the design and construction of distribution systems are done following power industry standards. The developed models are thus fairly generic and applicable to other utilities. The approach is also flexible. The failure rate models were derived using highresolution data but this does not prevent their application using input data as coarse as categorical storm conditions. Note that weather forecasting, especially high resolution forecasting, is a non-trivial effort but there are ongoing efforts for developing such technologies, e.g., the Deep-thunder system developed by
IBM [12]. This is beyond the scope of this study and not discussed further here.

The paper is organized as follows: Section II describes the high-resolution radar observations and utility outage data used in this study. Section III details the procedures of extracting and processing raw weather data from radar measurements to be used in the regression analysis for developing the failure rate model. In Section IV, the methodology to perform outage estimation and prediction using the FRMs is delineated by presenting the procedures to prepare the input weather data and testing of the failure rate models is performed. To overcome the issues of using the FRMs alone, a Bayesianbased approach is developed for predicting outages by following a non-homogeneous Poisson process (NHPP) in Section V. Section VI demonstrates the performance of this Bayesian update scheme and its potential benefits. Section VII summarizes this study and proposes the future research work.

\section{RADAR OBSERVATION AND GRID OUTAGE DATA}

\section{A. Weather Radar Data}

There are two types of weather data used in this research, radar data and local surface meteorological measurements from the National Weather Service (NWS) stations. Our study focuses on the service territories of two utilities in New York State (NYS). Fig. 1 shows one example of the radar observations (details to follow) plotted over the service territory areas.
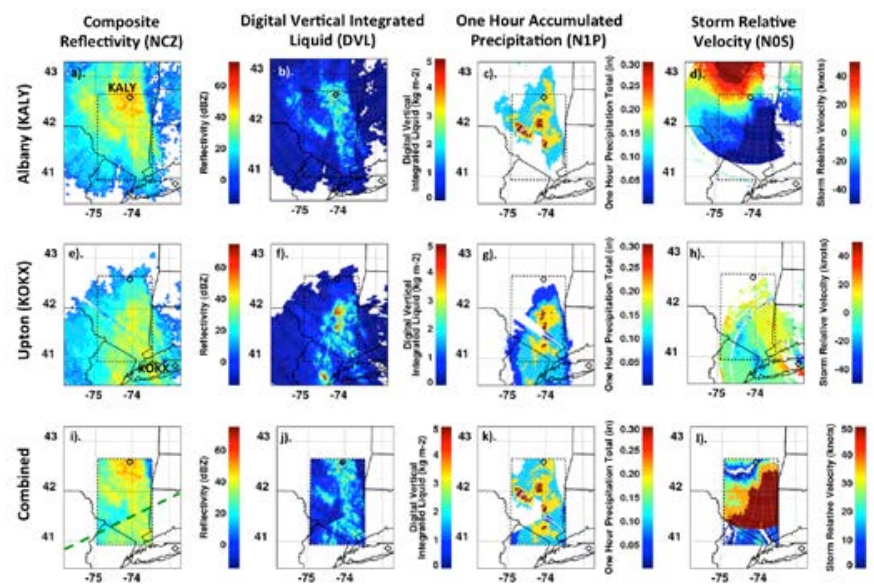

Fig. 1. Data for all four types of NEXRAD measurements (columns), row 1 KALY, row 2 - KOKX, and row 3, the combined fields.

Ten years (2005 through 2014) of historical weather radar data were obtained from two NWS Next-Generation Radar (NEXRAD) WSR-88D high-resolution Doppler weather radars [13] that provide primary coverage for these regions: Albany (KALY) and Upton (KOKX). The spatial and temporal resolutions of the radar observations are nominally 2$\mathrm{km}$ and 5-minute, respectively.

NEXRAD radars have a maximum coverage radius of 230 $\mathrm{km}$ (for radial velocity products), but provide optimal lowlevel coverage to within a $100 \mathrm{~km}$ radius; quality (low-level representativeness) degrades as the distance from the radar increases. The KALY and KOKX radars are located 
approximately $215 \mathrm{~km}$ from one another, with the dashed green line representing points equidistant from the radars. Observations to the northwest of this line will generally be collected from the Albany radar while those to the southeast will generally be from the Upton radar.

Four types of NEXRAD Level III products are used in the analysis, as listed in Table I [14]. These variables were chosen as representative of the severe weather signatures (large precipitation and high winds) most likely associated with power outages [15].

TABLE I: RADAR MEASUREMENTS OBTAINED FROM THE NEXRAD

\begin{tabular}{|l|l|l|l|}
\hline Symbol & Long Name & Description & Units \\
\hline NCZ & $\begin{array}{l}\text { Composite } \\
\text { reflectivity }\end{array}$ & $\begin{array}{l}\text { Maximum radar reflectivity in } \\
\text { each vertical column; describes } \\
\text { storm structure and intensity. }\end{array}$ & NBZ \\
\hline N0S & $\begin{array}{l}\text { Storm relative } \\
\text { velocity }\end{array}$ & $\begin{array}{l}\text { Produced by subtracting storm } \\
\text { motions from the general wind } \\
\text { field; helps identify rotation in } \\
\text { storms, which can be particularly } \\
\text { damaging. }\end{array}$ & Knots \\
\hline N1P & $\begin{array}{l}\text { One-hour } \\
\text { accumulated } \\
\text { precipitation }\end{array}$ & Hourly accumulated precipitation & Inches \\
\hline $\begin{array}{l}\text { *NVL, } \\
\text { DVL }\end{array}$ & $\begin{array}{l}\text { Vertically } \\
\text { integrated } \\
\text { liquid }\end{array}$ & $\begin{array}{l}\text { Water content of a 2.2 } 2.2 \mathrm{~nm} \\
\text { column of air; describes storm } \\
\text { intensity. }\end{array}$ & $\mathrm{kg} \mathrm{m}^{-}$ \\
\hline
\end{tabular}

* NVL was used prior to 2011, and DVL is used from 2011 forward.

Historical data were downloaded from the National Oceanic and Atmospheric Administration (NOAA) National Centers for Environmental Information website [16]. The downloaded data are processed in two steps. First, NOAA's Weather and Climate Toolkit is used in batch mode to convert each file in binary format to a NetCDF format file. The conversion places each of the data products on a horizontal grid with latitude/longitude spacing of $\sim 0.013^{\circ}$. (= 1.1 km $\left.-1.4 \mathrm{~km}\right)$. The next step is to take the NetCDF files from both radars, for a time step, for each variable (e.g., NCZ, NOS, etc.), and combine them into one horizontal field representing returns from both radars.

To combine these data from the two radars, we start by selecting a KALY file, and then find the KOKX file that complements it mostly closely in time, within 10 minutes. The grids of KALY and KOKX cover different regions; however, they are fixed for each data product from time step to time step. We adopt the KALY grid as the base grid, and the KOKX grid is transformed onto this grid via nearest-neighbor interpolation, within $.015^{\circ}$. For each spatial location, there are two possible values, the one from KALY and the one from KOKX. The value from the radar closest to the grid point is the default for the location, unless the value is missing. Equidistant locations are cross-compared for possible radar miscalibration or grid misalignment.

This selection approach is employed for composite reflectivity, vertically integrated liquid (VIL), and one-hour accumulated precipitation. However, for storm relative velocity fields, the maximum of the absolute value from the two radars is used. In rare cases when KALY data are unavailable for a timespan of at least two hours, KOKX values are used as the default and transformed onto the KALY grid.

\section{B. High Resolution Grid Outage Data}

The grid outage data were provided by two NYS utilities from their OMSs, from January, 2010 to September, 2014. The information for each outage includes a unique outage ID, a cause code, the coordinates of the component represented in terms of state plane, the type of the outage (whether the outage is underground or overhead), the times of outage occurrences and the repair of the outages, as well as the type of the components (e.g., an overhead distribution cable or a padmount transformer). Outages of forty-eight types of components were identified. However, the majority of these components are not modeled and their outages are not considered in this study ${ }^{1}$. Additional information may be provided, e.g., dispatch times and arrival times of repair crews.

The most important information to this study is the location of the outage/component, occurrence time, and the type of the component. These outages are not necessarily all caused by severe weather events. In general, if the outage occurrence time overlaps with a storm, the outages are considered to be storm induced. The outages that occur after a storm are excluded. It should be cautioned that the uncertainty associated with reported outages in an ongoing storm can be very large. The outage data acquired here are based on poststorm analyses, and can be considered close to a true report for the outages that actually happened during these events.

\section{Extracting Outage and Radar Measurement Weather Data}

It is recognized that, even under severe weather conditions, the occurrences of grid component outages can be very volatile, i.e., the correlation between weather conditions and outage is considered only statistically meaningful. On one hand, the smaller an area (e.g., a $2 \mathrm{~km}$ by $2 \mathrm{~km}$ area according the resolution of the radar measurements) is, the more uniform the weather conditions are expected in it. On the other hand, if the area is too small, there will not be an adequate number of grid components or outages in the area, which will make it difficult to achieve meaningful statistics for component failure rates. This results in a trade-off between the advantage of more uniform weather data (in a relative small area) and having a relative large number of grid components/outages (in a relatively large area). A two-step area radar data aggregation scheme was used in this study.

A region containing the service territory of one utility was selected in this study and divided into a number of smaller areas, e.g., 50 areas. The weather conditions across each small area should be relatively homogeneous. A very small area may have very few outages and components, which can diminish the benefit of using high-resolution weather data. Therefore, the 50 areas can be consolidated into a number of larger areas (as will be shown later.) Note that this does not mean that the

\footnotetext{
${ }^{1}$ Most of storm induced outages are overhead cable related. Other types of components include underground cables, group-operated air-break (GOAB) switches, capacitors, reclosers, sectionalizers, pad-mount transformers, fuses et al. The component information was extracted from a GIS (geographical information system) -based distribution system model.
} 
two-step aggregation must be used as one can simply aggregate the $2 \mathrm{~km}$ by $2 \mathrm{~km}$ areas into larger areas. It is expected that one additional step may remove some extreme measurement values and also makes it easier relatively easier to formulate different numbers of larger areas.

The following procedures were used to extract raw weather and outage data:

1)Identify a storm-induced outage of a component including its occurrence time, repair time, and location;

2)Identify the starting time and end time of the storm;

3)Identify the area containing the location in which this outage occurred;

4)Acquire radar data (defined Table I) and temperature data during the entire storm for the area;

5) Repeat steps $1-4$ to identify all other outages of the same type of components in different areas;

6) Repeat steps $1-5$ to identify other storm-induced outages;

7)Acquire inventory data of different types of components in different area.

These raw data were extracted using Matlab scripts and will be further processed and used for developing regression analysis based failure rate models, as will be shown in Section III.C. Step 2 was performed as follows: for each time step (approximately every 5 minutes), if there are more than 15 points within the entire domain with a reflectivity greater than $30 \mathrm{dBZ}$, or more than 7 points with a reflectivity greater than $40 \mathrm{dBZ}$, then the time step has storm activity. Radar reflectivity values of greater than $30 \mathrm{dBZ}$ are indicative of rainfall. A second pass is made to examine the times surrounding storm activity times ( $+/-45 \mathrm{~min})$, and where more than 6 points with greater than $30 \mathrm{dBZ}$ reflectivity are found, the storm identifier is made contiguous. A third pass is made and if there is a gap of less than a certain number of hours, e.g., two hours, between two storms, they are considered to be one storm.

\section{DeVELopment Of GENERALIZED LiNEAR REgRESSion BASED FAILURE RATE MODELS}

\section{A. GLM Based Regression Analysis}

Regression models are often used to describe a relationship between a response (characterized by a dependent response variable) and an observation consisting of one or more independent predictive terms (characterized by explanatory variables). This relationship can be linear or nonlinear. A special class of nonlinear models, called generalized linear models (GLMs), can be used to describe non-normal response distributions and modeling functions of the mean, and still be solved using linear methods [17]. A GLM consists of three components; a dependent random response variable of a certain type of distribution, a systematic component that specifies independent explanatory variables, and the link function of the mean of response variable that the model equates to the systematic component.

The failure rate of a component is always positive and the number of failures, or failure count data in the $i^{\text {th }}$-interval, is often described by a Poisson distribution, i.e., the number of failures $Y_{i} \sim \operatorname{Poisson}\left(\lambda_{i}\right)$ where $\lambda_{i}=E\left\{Y_{i}\right\}$ is the failure rate in the $i^{t h}$-interval. A GLM for component failure rate $\lambda_{i}$ using a logarithmic link function (assuming a Poisson failure process) is [7, 17]:

$$
\log \lambda_{i}=\log \frac{n_{i}}{N_{i}\left(t_{i}-t_{i-1}\right)}=b_{0}+b_{1} \bar{X}_{1}+\cdots+b_{p} \bar{X}_{p}
$$

where $n_{i}$ is the number of outages within the $i^{\text {th }}$-time interval $\left[t_{i-1}, t_{i}\right]$ among the total number $N_{i}$ of a specific type of component under weather conditions represented by $p$ variables $\bar{X}_{1}, \ldots, \bar{X}_{p}$. Equation (2) for predicting failure rates was used in this study:

$$
\log \lambda_{i}=b_{0}+b_{1} \bar{X}_{1}+b_{2} \bar{X}_{2}+b_{3} \bar{X}_{3}+b_{4} \bar{X}_{4}
$$

where the explanatory variable $\bar{X}_{1}$ represents radar reflectivity, $\bar{X}_{2}$ the accumulated precipitation, $\bar{X}_{3}$ the wind velocity, and $\bar{X}_{4}$ the temperature.

\section{B. Parameterization for Regression Analysis}

The following steps further illustrate the procedures in Section II.C and calculate the input to regression based failure rate model in Equation (2):

1)The region containing the utility's service territory is divided into $M_{a}$ small areas ( $M_{a}$ is chosen to be 50 in this study, i.e., $M_{a}=50$.)

2) Since radar observation is nominally available every 5minute, at a time instance $t_{j}$ when the measurements become available, the median value ${ }^{2}$. of weather data points in a small area $a_{i}$ is used to represent the weather condition of the area, and such data at each measurement time will be recorded until the outage occurs, which will formulate a weather data sequence corresponding to the outage.

The radar reflectivity data sequence for one of $M_{a}$ small areas $a_{i}$ in $\left[t_{0}, t_{N_{O}}\right]$ is given as:

$$
\begin{aligned}
& X_{1}\left(a_{i}\right)=\left[x_{1}\left(t_{0}, a_{i}\right) \quad x_{1}\left(t_{1}, a_{i}\right) \quad \cdots \quad x_{1}\left(t_{N_{O}}, a_{i}\right)\right] \\
& i=1,2, \cdots, M_{a}
\end{aligned}
$$

where $x_{1}\left(t_{j}, a_{i}\right)$ is the median value of all radar reflectivities (indicated by subscript 1 ) in area $a_{i}$ at time $t_{j}$ $\left(i=1, \cdots, M_{a}, j=0,1, \cdots, N_{O}\right)$ with $t_{0}$ representing the

\footnotetext{
${ }^{2}$ Because of the 2-km spatial resolution of radar measurement, a number of measurement points will be available at time $t_{j}$ in area $a_{i}$. There are different ways to synthesize a single set of weather condition data for an area, e.g., average of the weather data for this area or the maximum values. Medians of weather data were used here to avoid extreme values.
} 
starting time of the storm and $t_{N_{O}}$ the time when the outage occurs. It is naturally to assume that $x_{1}\left(t_{j}, a_{i}\right)$ will be a constant value within the time interval $\left[t_{j}, t_{j+1}\right]$.

3)A number of small areas are aggregated into a larger area $A_{k}, k=1,2, \cdots, M_{A}$, where the weather data sequence in this larger area is the average values of the data in all of the small areas in it given as

$$
\begin{aligned}
X_{1}\left(A_{k}\right)= & {\left[x_{1}\left(t_{0}, A_{k}\right) \quad x_{1}\left(t_{1}, A_{k}\right) \quad \cdots \quad x_{1}\left(t_{N_{O}}, A_{k}\right)\right] } \\
= & \frac{1}{M_{A}}\left[\sum_{a_{i} \in A_{k}} x_{1}\left(t_{0}, a_{i}\right) \quad \sum_{a_{i} \in A_{k}} x_{1}\left(t_{1}, a_{i}\right) \cdots\right. \\
& \left.\sum_{a_{i} \in A_{k}} x_{1}\left(t_{N_{O}}, a_{i}\right)\right]
\end{aligned}
$$

where each area $A_{k}$ consists of, e.g., 10, small areas, i.e., areas $a_{k_{1}}, a_{k_{2}}, \cdots, a_{k_{10}}$.

4) Definition of explanatory variables and parameterization:

a. Radar reflectivity, precipitation (DVL is not used since it covaries with precipitation), and temperature are selected in all analyses. Wind velocity is used for overhead component models but not for underground outages for obvious reasons;

b. In the radar reflectivity sequence data, the explanatory variable takes the weighted average value of all data points exceeding a pre-selected threshold, i.e., $20 \mathrm{dBZ}$ in this study, where the weight for a specific data point is the fraction of time this reflectivity remains over the entire time of the data sequence. The weighted average of the reflectivity data in one of the ten large areas $A_{k}$ is given in Equation (5) and will be directly used as one input in the right side of Equation (2):

$$
\begin{array}{r}
\overline{X_{1}}\left(A_{k}\right)=\frac{1}{t_{N_{O}}-t_{0}} \sum_{j=0}^{N_{O}}\left(t_{j+1}-t_{j}\right) x_{1}\left(t_{j}, A_{k}\right) \\
\forall x_{1}\left(t_{j}, A_{k}\right) \geq 20 d B
\end{array}
$$

5)For other weather condition data, the weighted average values are based on the data points corresponding to the reflectivity values exceeding the reflectivity threshold.

6)Hourly temperature data were obtained from a limited number of surface meteorological stations but will be used across the entire service territory since temperatures should not change dramatically in different locations.

The number of small areas, i.e., 50, is arbitrarily selected. So is the number of 10 larger areas or groups. These choices here are only for illustrative purpose and are subject to changes with more studies.

For procedure 4).b, the weighted average values of weather data are used because the likelihood of a component failure is determined by both: (1) how bad the weather conditions are, and (2) how long the component was exposed to such weather conditions. This is a unique feature of this study and needs to be highlighted.

Once the weighted average data for reflectivity, precipitation, wind velocity, and temperature were obtained for each outage, as shown in Steps 4 and 5 above, the GLM model in Equation (2) will be solved to estimate $b_{0}, \ldots, b_{4}$, which was done using the Matlab Statistical Toolbox. The regression models for the overhead $(\mathrm{OH})$ cable and overhead transformer components are shown in Table II, which can be directly used in (2) to calculate the failure rates, if weather condition data are given. Equipment failure rates for $\mathrm{OH}$ cables and transformers are in terms of the number of failures per mile per hour and number of failures per hour, respectively.

TABLE II: PARAMETERS FOR POISSON REGRESSION FAILURE RATE MODELS

\begin{tabular}{|c|l|l|l|l|l|}
\hline Components & $b_{0}$ & $b_{1}$ & $b_{2}$ & $b_{3}$ & $b_{4}$ \\
\hline $\begin{array}{c}\text { Overhead cable, } \\
\text { primary }\end{array}$ & -8.469 & -0.0026 & -5.77 & 0.068 & 0.1132 \\
\hline $\begin{array}{c}\text { Overhead, } \\
\text { Transformer }\end{array}$ & -12.5 & -0.085 & -0.93 & 0.085 & 0.083 \\
\hline
\end{tabular}

Regression models for additional components were also calculated, but not provided. This is because of the observations that (1) most or almost all of the outages are $\mathrm{OH}$ cable related and (2) the dependency of other equipment failure rates on weather conditions is not very strong. This is consistent with expectations since the susceptibility of wires to strong wind is well-known and failure mechanisms of other kinds of components, e.g., a pad mount transformer, remain to be better understood. This type of component may only be more susceptible to much stronger storms. However, such storms are rare and outage data (and/or radar data) for these cases are scarce. This is the major reason that this study focuses on distribution wires.

\section{WEATHER INPUT DATA PREPARATION AND FAILURE RATE Model Based OUTAGE PREDICTION}

Once the FRMs that link the outages and weather condition data are derived, the FRMs can be used to predict outages using the (forecasted) weather data ${ }^{3}$ of a specific storm. It is possible to use high-resolution data driven FRMs to predict outages at more refined temporal and spatial levels, i.e., from the beginning to the end of a storm or within an interval of a storm in a relatively small area. This section demonstrates how to prepare data for FRMs and how to estimate and predict outages using the FRMs and input data. Since forecasting is out of the scope of this study, this section describes how to prepare input using the actual radar measurement data and use them (with/without deviations) for the estimation and prediction, assuming that forecasted data have the same contents as the radar measurements.

\section{A. Weather Input Data Preparation and Outage Estimation}

The input data to the FRM only based outage estimation includes (1) radar measurements that were used to derive the weighted average values of weather data to represent the weather condition in a certain area within the selected time

\footnotetext{
${ }^{3}$ Forecasted weather data mean the forecasts of reflectivity, precipitation, wind speed, and temperature that were used to derive the FRMs.
} 
window of interest, and (2) the inventory of various components in the area, which is given for a utility system. The input data were prepared following the procedures in Section III.B with a time interval of one hour until the end of the storm. Note that this one-hour interval is chosen for illustration purpose only and will be used through the entire paper. Failure rates were calculated using the hourly weather condition data and used to calculate the number of failed components in the area on an hourly basis in this test, as discussed below.

Assuming an exponential failure distribution, the failure probability within time period $\left[T_{l}, T_{l+1}\right]$ of a particular type of component with a failure rate of $\lambda_{1}$ is given as:

$$
\operatorname{Pr}\left(T_{l} \leq t \leq T_{l+1}\right)=1-\exp \left(-\lambda_{l}\left(T_{l+1}-T_{l}\right)\right)
$$

If the total number of the functioning components is $N\left(T_{l}\right)$ at $t=T_{l}$, then the number of components that fail within this time period can be approximated as:

$$
N_{F}\left(T_{l+1}-T_{l}\right)=N\left(T_{l}\right) \times\left(1-\exp \left(-\lambda_{l}\left(T_{l+1}-T_{l}\right)\right)\right)
$$

and the number of components that remain functioning is:

$$
N\left(T_{l+1}\right)=N\left(T_{l}\right) \times \exp \left(-\lambda_{l}\left(T_{l+1}-T_{l}\right)\right)
$$

Theoretically, the development of a storm can be divided into an arbitrary number of time intervals. The procedures described using Equations (6) - (8) can be applied iteratively to update the number of component outages during a storm based on the available weather condition data forecast.

The OH cable FRM developed in Section III was used to estimate component outages based on actual radar measurement data. The hourly input data was prepared using Equations (3) - (5) to calculate failure rate $\lambda_{1}$ within $l^{\text {th }}$ interval and the hourly outage numbers were estimated using Equations (6) - (8).

The first case was performed for Hurricane Irene in August 2011, as shown in Fig. 2. In this storm, the total number of outages exceeded 400 , and most of them are $\mathrm{OH}$ cable related.
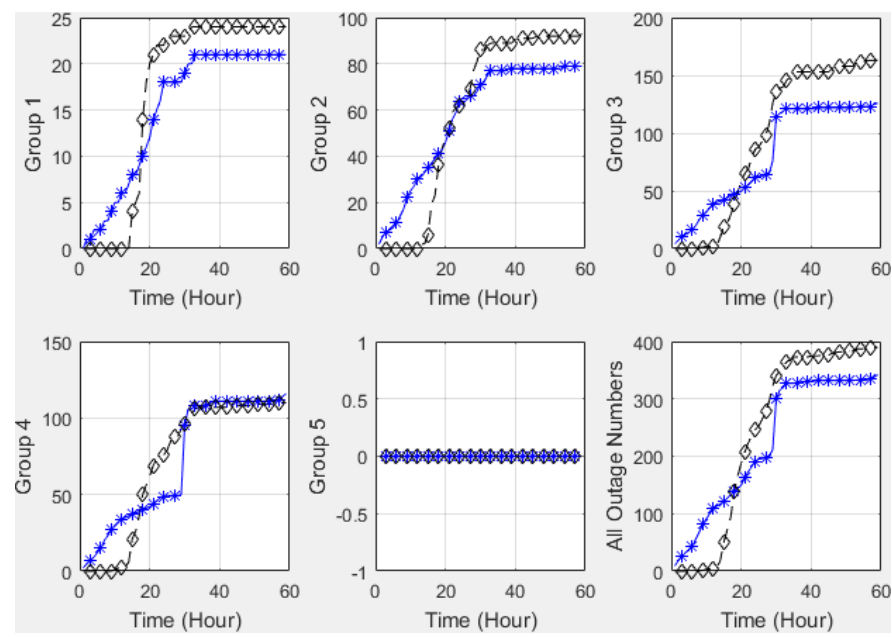

Fig. 2. OH cable outage calulation using failure rate model only for Hurrican Irene (diamonds for actual outages and stars for estimated ones).
In Fig. 2, the outages are shown for five separate aggregated areas (or Groups) of the same size (the detailed aggregation scheme is not shown due to a limit in the space but should not affect the results here since the algorithm is independent of the aggregation scheme) from the 50 smaller areas of the radar-covered region containing the utility service territory, plus the total outages in the last plot. As the storm became stronger and the number of outages increases, Fig. 2 shows that, even in smaller areas or groups, the trajectory of the estimated outages follows the actual outage evolution relatively closely, despite the volatile nature of the outage occurrences under storms and that each prediction in one-fifth of the entire area. In particular, the sum of estimated outages for different components is very close to the actual outages at the end of the storm (the deviation from the actual number of outages is around $10 \%$ ), as indicated in the last plot of Fig. 2. In addition, the trend in the outage number with time, while the storm is impacting the service area, is well captured. It should also be noted that the predicted outage numbers become relatively constant, especially for Groups 1 and 3 around Hour 30. This is mainly because of the $20 \mathrm{dBZ}$ reflectivity threshold, as indicated in Section III.B.

\section{B. Outage Predictions Using Pseudo Weather Forecasting}

Real-time high-resolution forecasting of weather conditions $[18,12]$ is outside the scope of this work. However, the forecasted weather conditions from numerical weather prediction models will certainly be less precise than analysis based on radar measurements used in the previous tests. Therefore, another set of tests were performed to predict outages assuming that forecasted weather data are available and can be represented by current radar data, but attributing different level of uncertainties for individual weather conditions. This is accomplished by adding perturbations to each of the different weather conditions separately, e.g., $\pm 70 \%$ on reflectivity, within the next forecasting time window.
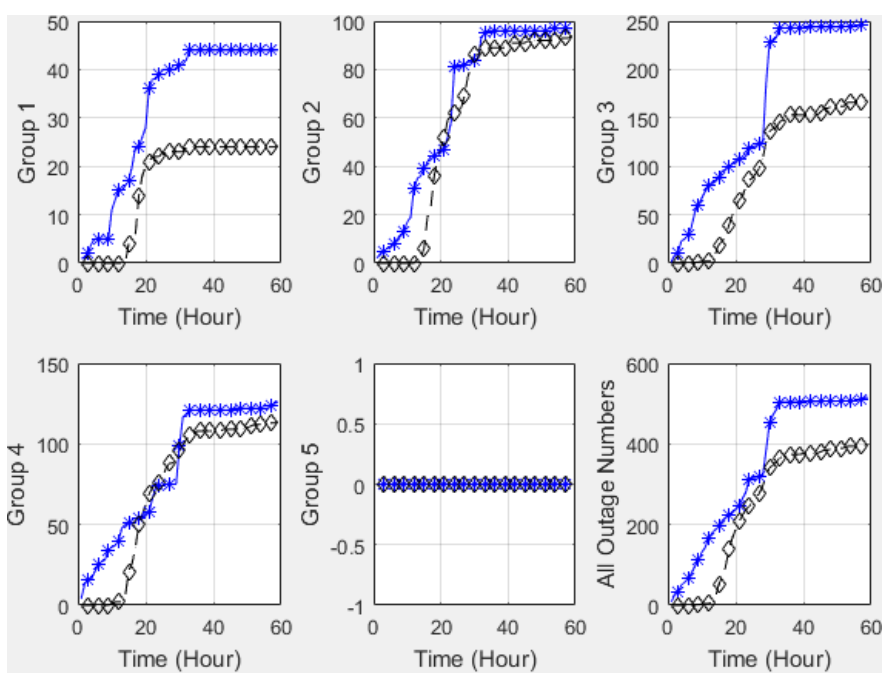

Fig. 3. OH cable outage prediction using the failure rate model and the pseudo forecasting data for Hurrican Irene (diamonds for actual outages and stars for predicted ones).

In Fig. 3, the perturbations of $\pm 70 \%$ were added to the each of the weather variables. Such data were again fed into the $\mathrm{OH}$ cable failure rate model to predict the number of outages that 
will occur in next time window. Fig. 3 shows an obvious increase in errors of outages in each of the five areas as well as the sum of outages. However, the trend of outage variation caused by weather condition evolution is still captured in Fig. 3.

\section{AN IMproved OUtAge Prediction SCHEME BASED ON THE BAYESIAN UPDATE}

The outage prediction using pseudo weather forecasting data in Section IV.B indicates that the inherent uncertainty in forecast weather variables results in much less precise outage prediction when using the developed failure rate models only. These weather forecast errors will also increase significantly as the forecasting time horizon is longer, magnifying the problem. Also, additional testing for mild storms (results are not shown here) indicates that outage prediction solely based on failure rate models may have large errors. Moreover, despite the large uncertainty associated with the real-time OMS reported outages (as indicated in Section II.B), such information may provide certain value to the outage prediction. This is particularly vital if the reported outages can be made more accurate through the installation of more sensing devices such as smart meters and communication links in the system. Therefore, development of an approach that can address all of these issues is crucial for a better prediction.

A Bayesian approach is adopted by using both failure rates estimated from weather data and the known/reported outage numbers during the storm. The rationale behind this selection of a Bayesian approach is that it is capable of accounting for confidence levels or beliefs on both strength and uncertainties of failure rate models and the reported outage information. The predicted failure rates provide a baseline for outage prediction and possess uncertainties for obvious reasons. There also exists a significant amount of uncertainty with the outage reporting in many different aspects, e.g., the timeliness, the location accuracy, the numbers, which are further exacerbated by the unavoidable issues with nested outages [11]. These uncertainties may be reduced by using both pieces of information in the prediction scheme.

\section{A. The Bayesian Approach}

A Bayesian approach consists of three steps: (1) the selection of prior distributions, (2) construction of likelihood functions, and (3) estimation of the posterior distributions using Bayes' theorem [19]. The prior distributions selected can be very diffuse. However, as more evidence becomes available, the posterior will become more and more concentrated, i.e., the uncertainty becomes smaller. The Bayesian estimation of a failure rate is given as:

$$
f_{\text {post }}(\lambda(t)) \propto \text { likelihood }(\lambda(t)) \times f_{\text {prior }}(\lambda(t))
$$

where $f(\cdot)$ represents a certain distribution for the failure rate and $\propto$ means "proportional to". Depending on the type of assumption about the failure process, $\lambda(t)$ can be timevariant for a non-homogeneous Poisson process (NHPP) or a constant for a homogeneous Poisson process (HPP).

\section{B. The Bayesian Update for Outages Following a Non- homogeneous Poisson Process}

As the weather conditions constantly change during a storm, so do the failure rates. Therefore, the NHPP assumption is much more reasonable. The NHPP has been applied extensively in describing the failure process for both hardware and software failures. The NHPP processes can be continuous (with continuous time variables) and discrete (with discrete time intervals).

Starting from time 0 , let $M(t)$ be the number of failures between time 0 and time $t$, and assume that $M(t)$ is described by an NHPP with mean $\Lambda(t)=E\{M(t)\}$. Therefore, the failures between different time intervals are independent and the mean for the number of failures between $T_{l}$ and $T_{l+1}$ is given as $\Lambda\left(T_{l+1}\right)-\Lambda\left(T_{l}\right)$ [20].

The likelihood function for observing no failures, one failure, or more failures at different times, is given in [10] and is not shown here. Assuming that $\Lambda_{t}=\Lambda(t)$ is of a gamma distribution given by $\Gamma\left(c \Lambda_{0}(t), c\right)$, therefore, $E\left\{\Lambda_{t}\right\}=$ $\Lambda_{0}(t)$, i.e., the prior belief on $\Lambda(t)$, the accumulated number of failures at time $t$. According to [14], the posterior distribution of $\Lambda(t)$ at $T_{l+1}$ is also a gamma distribution given by $\Gamma\left(c \Lambda_{0}(t)+\sum_{m \mid \tau_{m} \leq T_{l+1}} d_{m}, c+1\right)$, i.e., the updated mean accumulated number of failures is analytically given by

$$
\frac{c \Lambda_{0}(t)+\sum_{m \mid \tau_{m} \leq T_{l+1}} d_{m}}{c+1}
$$

where $d_{m}$ is the number of multiplicities of the failures observed at time $\tau_{m}, m=1,2, \cdots$, in the $m^{\text {th }}$ ordered epoch of the observed failures until the end of the failure process. Note that parameter $C$ indicates the confidence level on the prior belief for $\Lambda(t)$. A smaller $c$ means the actual number of observed failures plays a more important role and when $C=0$, the update is all determined by the new evidence of failures.

The storm is divided into $e$ epochs or intervals from the beginning of the storm, i.e., $\left[T_{0}, T_{1}\right], \ldots$, and $\left[T_{e}, T_{e-1}\right]$. The service territory is divided into $M_{a}$ small areas first and later aggregated into $M_{A}$ larger areas. The following Bayesian based algorithm is used to perform the outage prediction:

Algorithm 1: A Bayesian Outage Prediction (BOP) Algorithm

Given $N\left(T_{0}, A_{k}\right)$ and $\Lambda_{0}\left(T_{0}, A_{k}\right)=0$.

For $m=1,2, \ldots$, until $e$ :

For $k=1,2, \ldots$, until $M_{A}$ :

a) Calculate the weather input $\bar{X}_{1}\left(A_{k}\right), \ldots, \bar{X}_{4}\left(A_{k}\right)$ in $\left[T_{m}, T_{m-1}\right]$ using Equations (3) - (5) and the forecasted weather sequence data;

b) Calculate $\lambda_{m}\left(A_{k}\right)$ using $\bar{X}_{1}\left(A_{k}\right), \ldots, \bar{X}_{4}\left(A_{k}\right)$, Equation (2), and Table II for the selected type of components; 
c) Calculate numbers of components that are predicted to be failed and to remain functioning at $T_{m}, N_{F}\left(T_{m}-T_{m-1}, A_{k}\right)$ and $N\left(T_{m}, A_{k}\right)$, using Equations (6) - (8);

d) Let prior $\Lambda_{0}\left(T_{m}, A_{k}\right)=\Lambda_{0}\left(T_{m-1}, A_{k}\right)+N_{F}\left(T_{m}-T_{m-1}, A_{k}\right)$ and calculate the posterior $\Lambda\left(T_{m}, A_{k}\right)=$

$$
\frac{c \Lambda_{0}\left(T_{m-1}, A_{k}\right)+\sum_{m \mid \tau_{m} \leq T_{m}} d_{m}}{c+1} ;
$$

e) Update $\Lambda_{0}\left(T_{m}, A_{k}\right)=\Lambda\left(T_{m}, A_{k}\right)$;

\section{End}

End

In Step d, the observed outage number $d_{m}$ in $\left[T_{m-1}, T_{m}\right]$ is 0 (not occurred yet). The BOP algorithm is used at the beginning of each epoch to predict the outage numbers at the end of the epoch until storm is over for areas of a utility's service territory. Note that the BOP can be directly used to outage estimation by replacing forecasted weather data with measurement data from radar.

\section{OUtage Prediction Using the NHPP-BAsed BAyesian UPDATE SCHEME AND POTENTIAL BENEFIT ANALYSIS}

This section demonstrates the performance of the NHPPbased Bayesian update scheme developed above. The NHPPbased Bayesian update was applied to the utility that is divided into ten aggregated areas or groups shown in Fig. 4. The area with radar observations is divided into 50 small areas. The utility's distribution network is also shown in Fig. 4. Every five small areas are aggregated into a group (delimited with thick lines). A total number of ten groups or aggregated areas are shown in Fig. $4^{4}$.

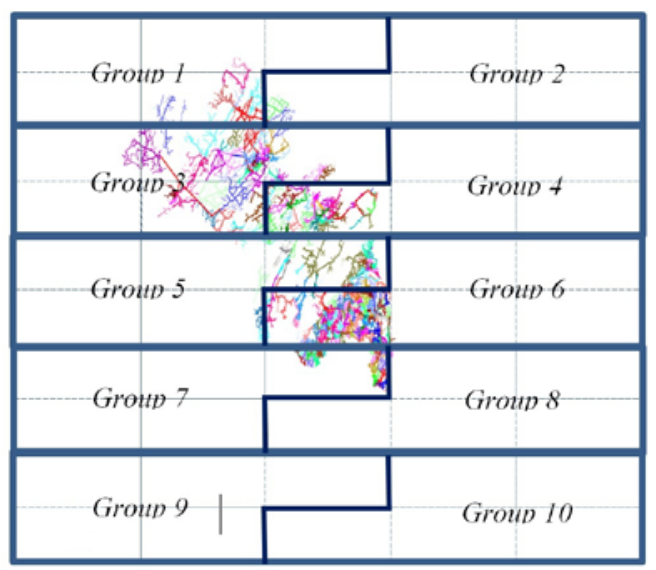

Fig. 4. Dividing the area containing the utility's system into ten groups.

The first test intends to show that the NHPP-based Bayesian update can perform a one-step-ahead prediction very well compared to the results where only failure rate models were used. The calculated $\mathrm{OH}$ cable outage numbers based on the NHPP-based Bayesian scheme are plotted together with the actual outage numbers against the storm hours for ten aggregated areas or groups containing the utility's distribution system shown in Fig. 4. Here, $C=0.5$, which means that the reported outages have more weight in the prediction. The

\footnotetext{
${ }^{4}$ Note that Groups 8 to 10 do not contain any distribution network. The areas were divided and aggregated in this way was because that weather data were obtained and processed first. This is not the best way to divide the service area and may increase the prediction errors.
}

weather forecasting uncertainties for the next hour can be up to $\pm 30 \%$, i.e., the pseudo-forecast was used by adding random uncertainties of up to $\pm 30 \%$ to the actual radar measurement data. Fig. 5 shows that the one-hour-ahead outage prediction in all ten groups is extremely accurate (diamonds for actual outages and stars for predicted ones.) However, it has to be pointed out that in Fig. 5, the original outage information was used as the reported outage data, i.e., we know exactly how many outages occurred before the prediction is made in each of the ten aggregated areas. As indicated in the previous section, this level of accuracy is impossible, especially given the extremely limited number of sensing devices and communication links installed in most of the distribution utilities at present.
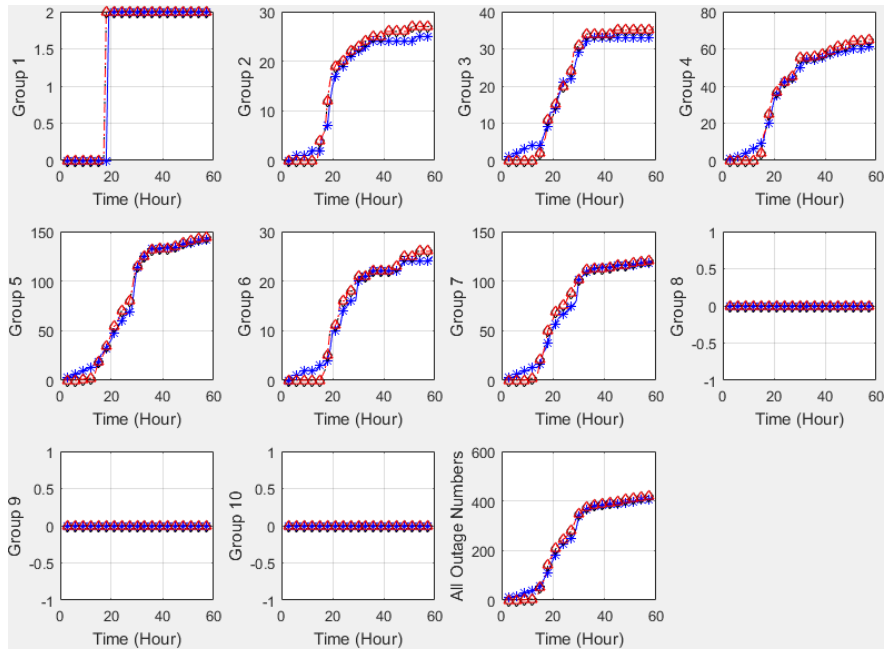

Fig. 5. OH cable outage prediction for Hurrican Irene, 2011 ( $c=0.5$, diamonds and triangles for actual and reported outages [the same], and stars for predicted ones.)

The next test case still uses the reported outage information. However, this outage information reported during the storm was obtained by adding a random disturbance up to $\pm 50 \%$ shift of the occurrence time of each recorded outage. The outage information reported was also including a random disturbance up to \pm 2 hours to the occurrence of outages for a particular type of components (overhead cables here) accumulated in each hour of the storm. Here, the value of parameter $C$ is chosen to be 1.2 and the uncertainties of the weather forecasting data are the same as the previous test case. The testing result is shown in Fig. 6.

In Fig. 6, the predicted outage curve (in stars) still lies between the actual (diamonds) and reported outages (triangles), but the curve leans more toward the calculated outages using failure rate models only $(c=1.2)$. Also, the calculated curve fits much better with the real outage curve by using the Bayesian update, compared to the case using the failure rates models only. This demonstrates the usefulness of the Bayesian update scheme. By choosing different values for $c$, one can give different weight to failure rate model based prediction and reported outage data. Note, if $C$ is chosen to be larger, then the predicted outage number will be much closer to the one calculated using failure rate model only. 

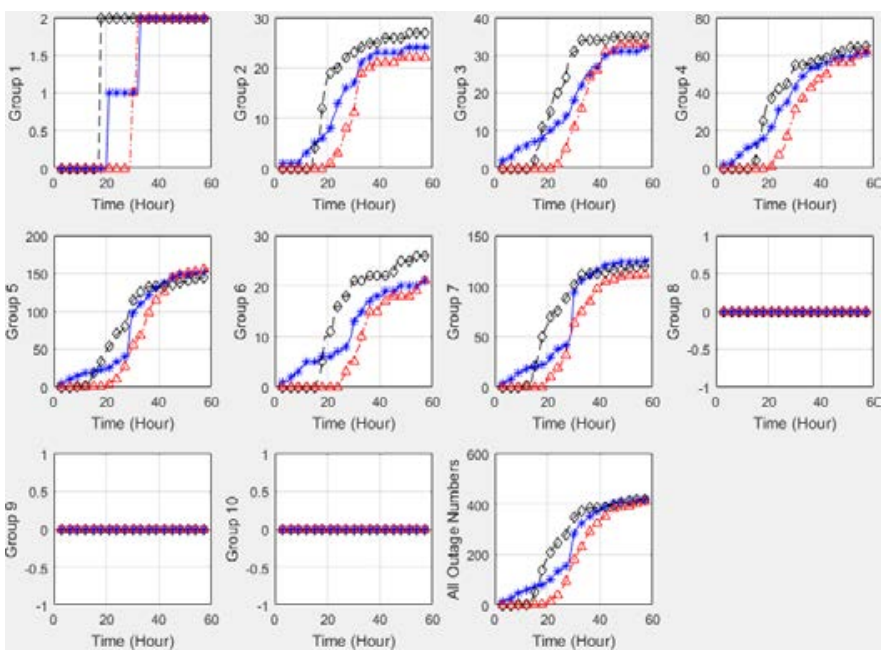

Fig. 6. OH cable outage prediction for Hurrican Irene $(c=1.2$, diamonds, triangles, and stars for actual, reported, and predicted outages, respectively.)

The $\mathrm{OH}$ transformer outage prediction during Hurricane Irene is shown in Fig. 7, where the same assumptions on the uncertainties and the same parameter $c$ were used. The total number of actual $\mathrm{OH}$ transformer outage (diamonds) is only 19. It is a challenge to predict outages in each of the 10 groups. However, Fig. 7 shows still that the prediction (stars) falls between the actual and reported ones (triangles) and the total number of predicted outages fits well with the actual outage along the development of the hurricane.
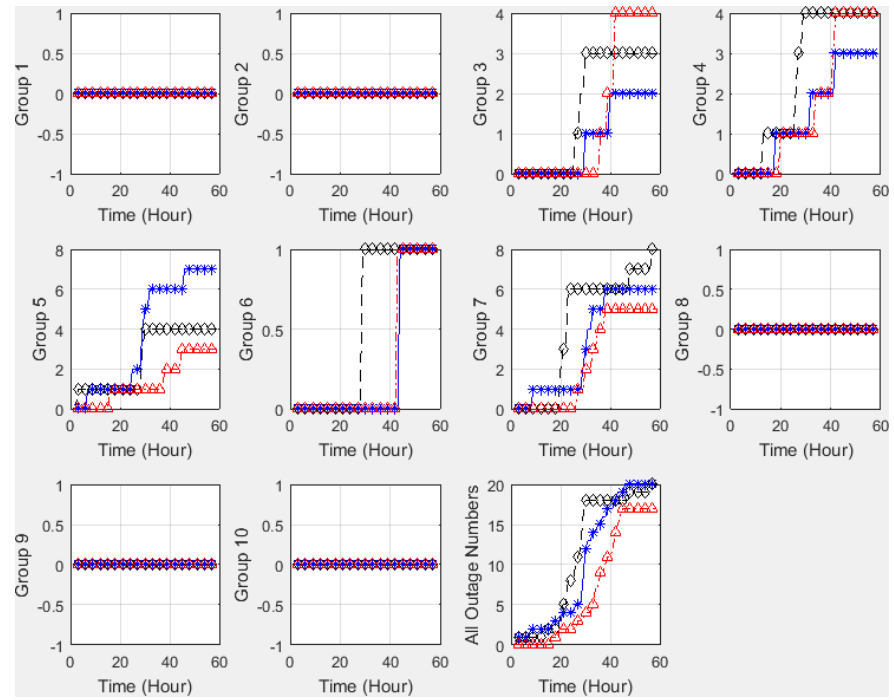

Fig. 7. $\mathrm{OH}$ transformer outage prediction for hurricane Irene $(c=1.2$, diamonds, triangles, and stars for actual, reported, and predicted outages, respectively.)

The next case study is based a storm that occurred in June of 2011. The storm lasted around 10 hours and was a fairly mild one since the total number of outages was only 27 across the entire utility service territory. The uncertainties associated with pseudo forecasted weather data and the outage numbers and their occurrence times are the same as above. The parameter $c$ was chosen as 2.0. The one-hour prediction of outages in each of the 10 groups is shown in Fig. 8.

As can be seen in Fig.8, in most of the groups the predicted outages (stars) still fall between the actual (diamonds) and reported ones (triangles). However, it is observed that predicted outages in Group 7 differ significantly from the actual and reported ones. This is mainly because that the FRMs work better for more severe events, as indicated previously. It is also noticed that the summation of outage numbers in different groups fits well with the actual outages (not surprisingly due to the statistical nature of the method), although the reported outage numbers are far less than the actual ones. In this case, if only the failure rate model is used or the parameter $c$ is chosen larger (not shown here), the prediction fits the actual outage evolution better because of large errors of reported outage numbers. However, this is considered more as a coincidence rather than a general property. Still, the results shown here demonstrate the usefulness of the proposed method, i.e., both FRMs and reported outage data play a role in the outage prediction while the proposed method enables their importance to be accounted for.
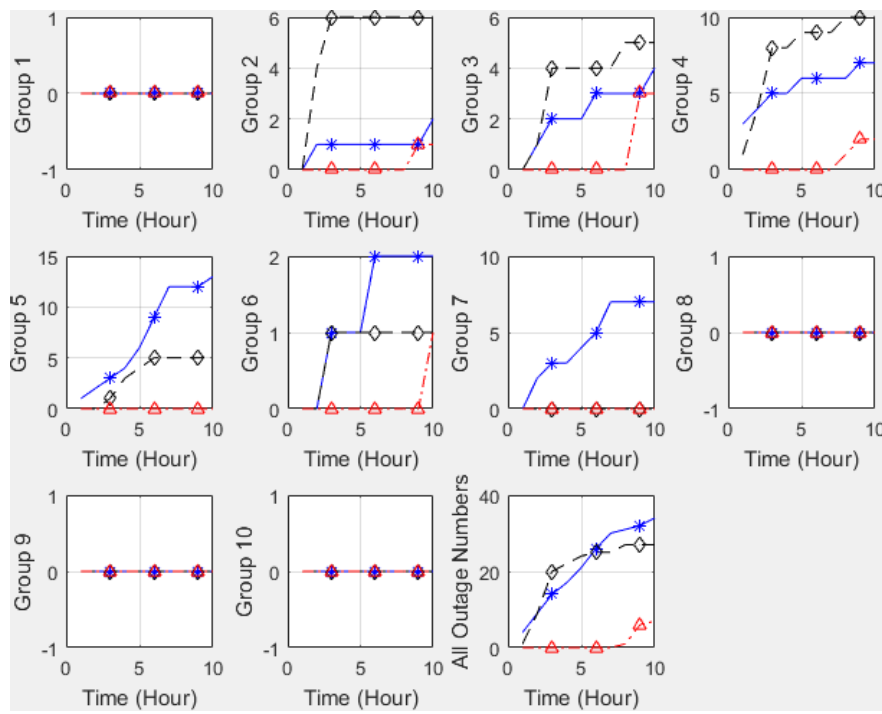

Fig. 8. OH cable outage prediction for a storm in June, $2011 \quad(c=2.0$ diamonds, triangles, and stars for actual, reported, and predicted outages, respectively.)

In the utility system of Fig. 4, the density of components in Group 7 of Fig. 4 is much greater than other groups. Therefore, Group 7 normally may have many more outages (although zero outages in Fig. 8). The utility would generally anticipate a need of more crews for storm restoration in Group 7. However, the outage numbers are proportional to both the total number of components and the severity of weather conditions that are location specific. In Fig. 6, although Group 2 contains only a small portion of the utility system compared to Group 1, the number of outages in Group 2 is much larger than that in Group 1. Similarly, a small number of outages occurred in Group 6 although Group 6 has a significant portion of the utility system. Thus, the information provided in Figs. 6, 7, and 8 can give a better picture of the outage distribution in the system within the forecasting time window. The radar measurement based outage prediction could have been used to position more crews to areas where more outages need to be fixed. This strategic positioning of the crews can reduce the storm restoration time and cost.

Because of the usage of high spatial and temporal resolutions of weather data in the FRM development, it is expected that one can predict outages at a more refined level, 
i.e., by dividing the service area into a larger number of groups instead of 10 or 5, as in this study. Again, one needs to caution about the appropriate sizes of groups or aggregated areas to consider the trade-off between uniformity of weather conditions and inventories of grid components, as indicated in Section II. It should also be noted that the sizes of the groups or aggregated areas do not have to be the same. Utilities can divide their service territory as needed, e.g., based on terrains or weather patterns. The proposed method can be used as long as inventory numbers of different types of components are known in each of the aggregated areas. This can be easily extracted if a GIS-based grid model is available.

\section{SUMMARY AND FUTURE WORK}

The first major contribution of this study is the development of regression models for failure rates of grid components based on a scheme that is capable of taking advantage of high resolution radar observations and grid component outage data. The radar data provide not only the peak values of weather conditions, which is what is typically used in other studies, but also how long grid components are exposed to these different peak values. The high-resolution data enables the calculation of the weighted average values of a set of peak values in weather condition data (i.e., reflectivity data points exceeding a certain threshold) that should have different impacts based on the duration of these values in the time window of interest (for outage estimation purpose) or the time it takes for a component to fail (as observations for the regression model development). This unique feature is the major difference between this work and other relevant studies.

The second major contribution is the development of a BOP algorithm to make use of both the failure rate models and the available reported outages during a severe storm. As discussed, the major issue with outage reporting in an ongoing storm is the potentially large uncertainties associated with the reported information. This information, however, may be used to help improve the outage prediction accuracy. Depending on the quality of the reported data, one can change the weights in the Bayesian update for failure rate models and the reported outage information.

GIS information or locational information has been used in the failure rate model development by considering variations of weather conditions and the number of different types of components in different areas. Longitude and latitude were provided in each snapshot of radar measurement. Therefore, the proposed method here can be implemented in Distribution Management System (DMS) or OMS provided that GIS information of grid components is available.

In addition, the proposed method for FRM development can be extended to capture the correlation between grid outages and other severe weather events such as ice storms, lightning strikes, flooding, or even damage assessment in other industries such as damage assessment of oil and gas pipelines upon the availability of the relevant data. The BOP algorithm provides a framework to combine the strength of both predictive methods and known outage data during severe weather events.
There is only one parameter that has to be chosen in the Bayesian update, i.e., the belief we have $c$ on the prior information. This parameter decides the importance of prior information and the newly observed failures in the Bayesian update. Therefore, the parameter may sound subjective but actually can be better determined with more actual data to test and improve the accuracy of FRM based outage estimation and the accuracy of reported/inferred outages in real time. This will be further investigated in the future.

A number of additional future research activities have been identified, in addition to the collection of more quality outage data, which include (1) a careful examination of outage data to identify outages that are more storm-relevant, whether the outage is secondary or not, and better understanding of storminduced failure mechanisms for different types of grid components; (2) an examination of areas of different sizes that can be considered to have uniform weather conditions; (3) different schemes to extract weather data to better represent the weather conditions in a pre-selected area; (4) modeling of factors that may have significant impacts on component failure such as vegetation or soil moisture [3, 5, 21] will be considered; (5) how to improve the accuracy of the outage reporting based on field measurement from different sensing devices, e.g., via inference of available smart meter data in distribution networks ${ }^{5}$. Furthermore, this proof-of-concept study used a limited amount of available outage data. Upon the availability of sufficient data, the FRMs for individual components may be universally applicable. The results demonstrated the potential benefits of having high temporalspatial resolution data of both external weather events and grid component outages, which may motivate the collection of granular data among distribution utilities. Furthermore, collection and application of more data will help improve the accuracy of FRMs.

It is suggested that the methodology developed herein be taken one step further to integrate it into a distribution planning tool and better understand the grid impacts caused by the outages of the different types of components. The component outage information indicates a component failure; however, it does not tell what impact that failure may have on the grid, i.e., loss of customers. To understand the grid impact of the outages, the use of a grid model together with the failure rate models should be evaluated to assess the grid impacts by providing the number of component outages in different aggregated areas and running simulations.

\section{REFERENCES}

[1] Y. Wang, C. Chen, J. Wang, and R. Baldick, "Research on Resilience of Power Systems under Natural Disasters - A Review,” IEEE Transactions on Power Systems, Vol. 31, No. 2, Mar. 2016.

[2] M. Allen et al, "Application of Hybrid Geo-Spatially Granular Fragility Curves to Improve Power Outage Predictions,” Journal of Geography and Natural Disasters, Vol. 4, Issue 2, 2014.

[3] R. Nateghi, S. Guikema, and S. M. Quiring, "Power Outage Estimation for Tropical Cyclones: Improved Accuracy with Simpler Models,” Risk Analysis, Vol. 34, No. 6, 2014.

\footnotetext{
${ }^{5}$ This is currently studied in an ongoing project funded by Department of Energy GMLC (Grid Modernization Laboratory Consortium) program.
} 
[4] S. Guikema et al, "Predicting Hurricane Power Outages to Support Storm Response Planning,” IEEE Access the Journal for Open Access Publishing, Vol. 2, 2014.

[5] S.-R. Han, S. Guikema, S. Quiring, K.-H. Lee, D. Rosowsky, and R. A. Davidson, "Estimating the spatial distribution of power outages during hurricanes in the Gulf Coast region,” Reliability Engineering and System Safety, Vol. 94, No. 2, pp 199-210, 2009.

[6] P.-C. Chen and M. Kezunovic, "Fuzzy Logic Approach to Predictive Risk Analysis in Distribution Outage Management," IEEE Transactions on Smart Grid, Vol. 7, No. 6, Nov. 2016.

[7] Y. Zhou, A. Pahwa, and S-S. Yang, "Modeling Weather-Related Failures of Overhead Distribution Lines," IEEE Transactions on Power Systems, Vol. 21, No. 4, Nov. 2006.

[8] F. Xiao, J. D. McCalley, Y. Ou, J. Adams, and S. Myers, "Contingency Probability Estimation Using Weather and Geographical Data for OnLine Security Assessment," 9th International Conference on Probabilistic Methods Applied to Power Systems, KTH, Stockholm, Sweden, Jun. 2006.

[9] Y. Liu and C. Singh, "A Methodology for Evaluation of Hurricane Impact on Composite Power System Reliability,” IEEE Transactions on Power Systems, Vol. 26, No. 1, Feb. 2011.

[10] D. Zhu, D. Cheng, R. Broadwater and C. Scirbona, "Storm Modeling for Prediction of Power Distribution System Outages," Vol. 77, pp 973-979, Electric Power Systems Research, 2007.

[11] M. Vadari, "Electric System Operations: Evolving to the Modern Grid," Artech House, November, 2012.

[12] L. Treinish et al., "On-going Utilization and Evaluation of a Coupled Weather and Outage Prediction Service for Electric Distribution Operations," in Proc. 4th Conf. Weather Climate New Energy Economy, Austin, TX, USA, Jan. 2011.

[13] T. D. Crum, R. E. Saffle, and J. W. Wilson, "An update on the NEXRAD program and future WSR-88D support to operations," Weather and Forecasting, Vol. 13, pp 253-262, 1998.

[14] K. E., Kunkel et al, "Monitoring and Understanding Trends in Extreme Storms: State of Knowledge,” Bull. Amer. Meteor. Soc., Vol. 94, pp 499-514, doi:10.1175/BAMS-D-11-00262.1, 2013.

[15] R. J. Doviak, and D. S. Zrnic, "Doppler Radar and Weather Observations,” Dover, pp 562, 1993.

[16] Available at https://www.ncdc.noaa.gov/data-access/radar-data.

[17] A. Agresti, "Categorical Data Analysis," A John \& Wiley Sons, Inc., Publication, 2002.

[18] Dustan M. Wheatley, Kent H. Knopfmeier, Thomas A. Jones, and Gerald J. Creager, "Storm-Scale Data Assimilation and Ensemble Forecasting with the NSSL Experimental Warn-on-Forecast System. Part I: Radar Data Experiments,” Weather and Forecasting, Vol. 30, No. 6, pp 1795-1817, 2015.

[19] C. L. Atwood et al., "Handbook of Parameter Estimation for Probabilistic Risk Assessment,” U.S. Nuclear Regulatory Commission, Tech. Rep. NUREG/CR-6823, Sep. 2002.

[20] L. Kuo and S. Ghosh, "Bayesian Nonparametric Inference for Nonhomogeneous Poisson Processes,” Technical Report 9718, University of Connecticut, Department of Statistics, 1997.

[21] T. Dokic, P.-C. Chen, M. Kezunovic,. "Risk Analysis for Assessment of Vegetation Impact on Outages in Electric Power Systems,." CIGRE US National Committee 2016 Grid of the Future Symposium, Philadelphia, PA, USA, Oct. 2016. 